\title{
Correction to Thermochemistry of Racemic and Enantiopure Organic Crystals for Predicting Enantiomer Separation
}

Hannes K. Buchholz, Rebecca K. Hylton, Jan Gerit Brandenburg, Andreas Seidel-Morgenstern, Heike Lorenz, Matthias Stein,* and Sarah L. Price*(1)

Cryst. Growth Des. 2017, 17 (9), 4676-4686. DOI: 10.1021/acs.cgd.7b00582

S Supporting Information

We apologize that eq $\mathrm{S} 10$ in our originally published Supporting Information needed a critical change of a to + sign. We also took the opportunity to improve eqs S14 and S15. There are no other changes. The corrected Supporting Information accompanies this Addition and Correction.

\section{ASSOCIATED CONTENT}

\section{S Supporting Information}

The Supporting Information is available free of charge on the ACS Publications website at DOI: 10.1021/acs.cgd.7b01381.

Additional information regarding computational details, materials and methods and experimental measurements of heat capacities and solubilities (PDF) 